\title{
A Novel Technique for the Construction of Safe Substitution Boxes Based on Cyclic and Symmetric Groups
}

\author{
Abdul Razaq $\left({ }^{1},{ }^{1}\right.$ Hanan A. Al-Olayan, ${ }^{2}$ Atta Ullah, ${ }^{3}$ Arshad Riaz $\left(\mathbb{D},{ }^{1}\right.$ and Adil Waheed ${ }^{4}$ \\ ${ }^{1}$ Department of Mathematics, University of Education Lahore, Jauharabad Campus, Pakistan \\ ${ }^{2}$ Department of Mathematics, King Saud University, Saudi Arabia \\ ${ }^{3}$ Department of Mathematics, Quaid-i-Azam University Islamabad, Pakistan \\ ${ }^{4}$ Department of Information Technology, University of Education Lahore, Jauharabad Campus, Pakistan
}

Correspondence should be addressed to Abdul Razaq; makenqau@gmail.com

Received 4 June 2018; Accepted 19 September 2018; Published 4 October 2018

Academic Editor: Stelvio Cimato

Copyright (c) 2018 Abdul Razaq et al. This is an open access article distributed under the Creative Commons Attribution License, which permits unrestricted use, distribution, and reproduction in any medium, provided the original work is properly cited.

\begin{abstract}
In the literature, different algebraic techniques have been applied on Galois field $G F\left(2^{8}\right)$ to construct substitution boxes. In this paper, instead of Galois field $G F\left(2^{8}\right)$, we use a cyclic group $C_{255}$ in the formation of proposed substitution box. The construction proposed S-box involves three simple steps. In the first step, we introduce a special type of transformation $T$ of order 255 to generate $C_{255}$. Next, we adjoin 0 to $C_{255}$ and write the elements of $C_{255} \cup\{0\}$ in $16 \times 16$ matrix to destroy the initial sequence $0,1,2, \ldots, 255$. In the $2^{\text {nd }}$ step, the randomness in the data is increased by applying certain permutations of the symmetric group $S_{16}$ on rows and columns of the matrix. In the last step we consider the symmetric group $S_{256}$, and positions of the elements of the matrix obtained in step 2 are changed by its certain permutations to construct the suggested S-box. The strength of our S-box to work against cryptanalysis is checked through various tests. The results are then compared with the famous S-boxes. The comparison shows that the ability of our S-box to create confusion is better than most of the famous S-boxes.
\end{abstract}

\section{Introduction}

The foundation of modern cryptography was laid by Shannon [1]. Cryptography is the science of converting the secret information into dummy data so that it could reach the destination safely without leakage of the information. The modern cryptography is divided into several branches. However, symmetric key cryptography and public key cryptography are the two main areas of study. In symmetric key cryptography, the same key is used at both ends to encrypt and decrypt data/information, but in public key cryptography two different keys, public and private keys, are used. It is well-known that, in symmetric key cryptography the substitution box is a standout and basic ingredient, which performs substitution. In block ciphers, it is widely used to make the relationship between the ciphertext and the key unclear and vague. Due to these important applications of substitution box many algorithms have been developed to construct safer and more reliable S-boxes. Substitution boxes are used for the strong design of block encryption algorithms. S-box is the only nonlinear component for most of the block encryption algorithms such as international data encryption algorithm (IDEA), advanced encryption standard (AES), and data encryption standard (DES) [2]. Substitution boxes yield a DES-like cryptosystem with the perplexity property depicted by Shannon. In [3], it is shown that for weaker S-boxes, DES can be easily broken. It means that the security of DES-like cryptosystems is merely determined by the quality of the S-boxes used. Thus, in order to develop secure cryptosystems, the formation of safe S-boxes is a main focus of the researcher. To examine the strength of S-boxes, nonlinearity test, bit independent criterion, strict avalanche criterion, linear approximation probability analysis, differential uniformity test, and majority logic criterion are used. In the literature, there are many S-box construction methods such as inversion mapping, power polynomial, heuristic methods, and pseudorandom methods [4]. Incursions on the S-box component of data encryption standard (DES) damage 


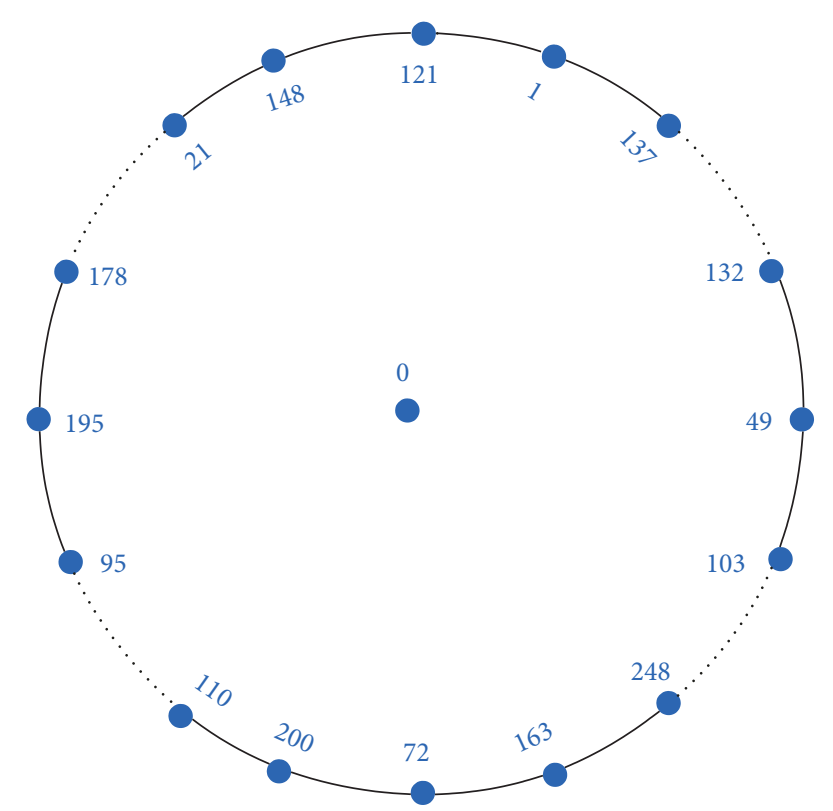

FIgURE 1: Cayley graph of $C_{255} \cup\{0\}$.

the design process of advanced encryption standard (AES) $[3,5]$. Therefore, the substitution box component of AES is designed to ensure the security of the data/information in the presence of differential and linear cryptanalysis attacks [6].

Recently, since proposed algebraic attacks have been succeeded in some loops of AES, researchers have focused on alternative construction methods for substitution box [21]. Therefore, substitution box construction techniques based on group theory have been applied for alternative substitution box designs.

\section{Algebraic Structure of Proposed Substitution Box}

Let us denote a set of positive integers less than 256 by $\Upsilon$; that is, $\Upsilon=\{1,2,3, \ldots, 255\}$. Consider a transformation $T: \Upsilon \longrightarrow$ $\Upsilon$ defined by

$$
\begin{aligned}
& T(z) \\
& \quad= \begin{cases}\left(\frac{z}{16 z+1}\right)(\bmod 257) & \text { if } z \in \Upsilon-\{16,136\} \\
\left(\frac{z}{32 z+1}\right)(\bmod 257) & \text { if } z=16,136\end{cases}
\end{aligned}
$$

It can be easily verified that $T$ has order 255; that is, for any $z \in \Upsilon, T^{255}(z)=z$. Thus for all $z \in \Upsilon, T(z)$ generates a cyclic group $C_{255}=\left\{T(z), T^{2}(z), T^{3}(z), \ldots, T^{254}(z), z\right\}$. In this paper, we have taken $z=1$.

Step I. First we simply present the elements of

$$
C_{255} \cup\{0\}=\left\{T(1), T^{2}(1), T^{3}(1), \ldots, T^{254}(1), 1,0\right\}
$$

in $16 \times 16$ matrix (see Table 2). Cayley graph of $C_{255} \cup$ $\{0\}$ is shown in Figure 1. In this way, the initial sequence $0,1,2, \ldots, 255$ is destroyed. If this matrix is conceded as Sbox, its nonlinearity is 103.75 , which is acceptable. Now we move to step II to create more randomness.

Step II. Since we have presented our data in $16 \times 16$ matrix, that is, a matrix with 16 rows and 16 columns, the randomness can be increased by interchanging the positions of the rows and columns. Algebraically, it is achieved by applying permutations of the symmetric group $S_{16}$ on the matrix. Since order of $S_{16}$ is 16 !, therefore corresponding to one matrix (S-box) formed after applying one permutation on rows, 16 ! number of new S-boxes can be created by applying all the permutations on columns. Thus by this technique, we can construct $(16 !)^{2}$ different S-boxes. We choose two particular types of permutations of the symmetric group $S_{16}$ such that one of them is applied on the rows and the other on columns. This action increases the diffusion capability of the cipher. The permutations are as follows.

(1) $(2,7,11)(3,6,10,13,5,8)(4,9,12)(14)(15)(16)$ : applied on rows 
TABLE 1: The permutation of $\mathrm{S}_{256}$ used in step 3.

\begin{tabular}{|c|c|c|c|c|c|c|c|c|c|c|c|c|c|c|c|}
\hline$(1$ & 225 & 221 & 169 & 78 & 255 & 136 & 173 & 62 & 146 & 56 & 119 & 229 & 114 & 117 & 174 \\
\hline 143 & 247 & 105 & 16 & 197 & 139 & 201 & 205 & 124 & 15 & 103 & 80 & 133 & 228 & 74 & 13 \\
\hline 166 & 127 & 226 & 53 & 219 & 181 & 209 & 45 & 251 & 60 & 43 & 232 & 160 & 239 & 71 & 9 \\
\hline 64 & 231 & 208 & 18 & 98 & 115 & 254 & 213 & 150 & 75 & 82 & 27 & 111 & 230 & 11 & 227 \\
\hline 184 & 30 & 212 & 241 & 248 & 170 & 17 & 235 & 32 & 249 & 207 & 77 & 69 & 95 & 252 & 81 \\
\hline 222 & 149 & 92 & 73 & 57 & 23 & 162 & 61 & 89 & 220 & 211 & 175 & 91 & 33 & 157 & 223 \\
\hline 68 & 159 & 14 & 54 & 83 & 191 & 193 & 0 & 102 & 24 & 90 & 183 & 126 & 116 & 134 & 37 \\
\hline 144 & 244 & 192 & 35 & 253 & 233 & 216 & 187 & 196 & 198 & 104 & 84 & 47 & 155 & 178 & 106 \\
\hline 34 & 128 & 101 & 206 & 50 & 148 & 94 & 245 & 19 & 93 & 40 & 97 & 171 & 165 & 125 & 189 \\
\hline 195 & 63 & 121 & 3 & 164 & 4 & 29 & 137 & 129 & 52 & 203 & 79 & 123 & 177 & 182 & 176 \\
\hline 39 & 96 & 215 & 238 & 67 & 107 & 210 & 25 & 179 & 141 & 242 & 31 & 243 & 41 & 8 & 200 \\
\hline 186 & 110 & 199 & 152 & 108 & 65 & 12 & 237 & 59 & 85 & 118 & 113 & 46 & 120 & 142 & 185 \\
\hline 20 & 147 & 190 & 28 & 36 & 153 & 140 & 5 & 135 & 99 & 21 & 49 & 7 & 38 & 188 & 55 \\
\hline 42 & 240 & 109 & 167 & 145 & 2 & 236 & 151 & 122 & 224 & 218 & 132 & 163 & 86 & 180 & 48 \\
\hline 131 & 194 & 88 & 10 & 26 & 156 & 246 & 168 & 214 & 100 & 58 & 6 & 66 & 204 & 22 & 130 \\
\hline 51 & 202 & 158 & 172 & 234 & 161) & $(44$ & 72 & 154 & 76 & 138 & 217 & 112) & $(70$ & 250 & 87) \\
\hline
\end{tabular}

The resulting S-box (see Table 3) has nonlinearity of 106.25. In step III, we further enhance its working capability.

Step III. Recently, we have noticed that certain permutations of the symmetric group $S_{256}$ are amazingly constructive. In this step, we apply a permutations of $S_{256}$ (see Table 1) on the data/matrix obtained after step II to construct a very strong S-box (see Table 4).

\section{Security Analysis}

In this section, a point by point exploration of the suggested $\mathrm{S}$-box is presented. Furthermore, we have made a comparison with the famous S-boxes, such as AES S-box, Xyi S-box, Skipjack S-box, S8 AES S-box, Residue Prime S-box, APA S-box, and Gray S-box. The illustration of various analysis applied on these substitution boxes is given. It is seen that our S-box meets all the standards near the ideal status.

3.1. Nonlinearity. The key objective of the substitution box is to provide assistance in giving nonlinear change from unique data to the encoded information. The measure of nonlinearity presented by the cipher considered as the most important part in the entire process of encryption. It is defined as

$$
N_{f}=2^{r-1}\left(1-2^{-r} \max \left|W_{(f)}(z)\right|\right) .
$$

Here

$$
W_{(f)}(z)=\sum_{z \in F_{2}^{r}}(-1)^{f(x) \otimes+\cdot z}
$$

is the Walsh Spectrum. The average values of the nonlinearity of newly constructed S-box is 112. A comparison between the nonlinearity of the suggested S-box and multiple renowned substitution boxes is given in Table 5 .
3.2. Bit Independence Criterion. Webster and Tavares firstly demonstrated bit independence criterion [22]. A function $h:\{0,1\}^{n} \longrightarrow\{0,1\}^{n}$ fulfils the BIC requirements if $\forall i, j, k \in$ $\{1,2,3, \ldots, n\}$, the output bits $j$ and $k$, where $j \neq k$, change independently by inverting the input bit $i$. In cryptographic systems, the BIC is a very important characteristic because by increasing independence between bits, it is very hard to decipher and predict the scheme of the system. The outcomes of nonlinearity of BIC are presented in Table 6. In order to find the independence properties a comparison of the bits, created by the eight basic functions, with each other is established. The relationship between the outcomes of change in ith input bit and the change in jth and kth output bits is identified. In the first phase the ith bit is varied from 1 to $n$ by keeping $j t h$ and $k t h$ bits fixed. Next, the values of $j$ and $k$ are altered from 1 to $n$. Furthermore, the minimum and average values of BIC along with square deviation of the proposed S-boxes are presented in Table 7. The average and minimum values of BIC of the proposed S-box are 112. The square deviation of the newly created substitution box is 0 . All these results are better than most of the well-known S-boxes and similar to AES, $\mathrm{S}_{8}$ AES, and Gray S-boxes.

3.3. Strict Avalanche Criterion Analytically. Tavares and Webster introduced strict avalanche criterion [22]. In this criterion, the output bits are examined after changing a single input bit. In ideal condition, by changing a single input bit, half of the output bits change their shape. In [23] an effective technique is presented to check whether a complete substitution box satisfies the SAC or not. The results of SAC of the suggested S-box (see Table 8 ) are nearly equal to $1 / 2$, which shows its strength.

3.4. Linear Approximation Probability. In this analysis, the imbalance of an event is examined. It is useful in finding the maximum value of an imbalance of the output in an 
TABLE 2: $16 \times 16$ matrix evolved after $1^{\text {st }}$ step.

\begin{tabular}{|c|c|c|c|c|c|c|c|c|c|c|c|c|c|c|c|}
\hline 121 & 148 & 21 & 87 & 165 & 53 & 116 & 2 & 39 & 174 & 106 & 4 & 91 & 8 & 16 & 241 \\
\hline 249 & 166 & 253 & 151 & 83 & 218 & 255 & 141 & 204 & 92 & 170 & 236 & 109 & 136 & 120 & 199 \\
\hline 175 & 102 & 244 & 46 & 44 & 85 & 133 & 118 & 47 & 183 & 221 & 68 & 157 & 128 & 202 & 60 \\
\hline 95 & 228 & 201 & 216 & 250 & 51 & 219 & 122 & 161 & 23 & 131 & 22 & 27 & 171 & 178 & 195 \\
\hline 40 & 59 & 247 & 152 & 224 & 220 & 144 & 239 & 52 & 34 & 84 & 207 & 167 & 64 & 150 & 101 \\
\hline 111 & 30 & 186 & 176 & 188 & 114 & 123 & 229 & 130 & 108 & 160 & 125 & 208 & 154 & 187 & 238 \\
\hline 6 & 61 & 24 & 209 & 245 & 140 & 98 & 194 & 254 & 11 & 138 & 142 & 35 & 214 & 180 & 89 \\
\hline 153 & 226 & 66 & 20 & 177 & 158 & 203 & 252 & 192 & 76 & 14 & 112 & 67 & 110 & 200 & 72 \\
\hline 163 & 248 & 169 & 26 & 164 & 17 & 242 & 42 & 73 & 232 & 78 & 212 & 182 & 32 & 225 & 75 \\
\hline 45 & 179 & 25 & 184 & 215 & 15 & 240 & 93 & 231 & 88 & 9 & 94 & 185 & 57 & 147 & 190 \\
\hline 145 & 243 & 181 & 65 & 5 & 54 & 99 & 80 & 237 & 191 & 31 & 104 & 168 & 77 & 43 & 222 \\
\hline 115 & 119 & 246 & 3 & 63 & 159 & 117 & 12 & 48 & 233 & 196 & 251 & 19 & 70 & 103 & 49 \\
\hline 132 & 97 & 149 & 127 & 28 & 134 & 143 & 69 & 81 & 71 & 227 & 146 & 156 & 107 & 193 & 90 \\
\hline 50 & 173 & 223 & 205 & 18 & 113 & 37 & 33 & 105 & 10 & 198 & 217 & 62 & 79 & 86 & 230 \\
\hline 235 & 126 & 234 & 96 & 135 & 38 & 206 & 7 & 41 & 56 & 29 & 162 & 197 & 55 & 129 & 100 \\
\hline 189 & 36 & 74 & 210 & 139 & 124 & 172 & 213 & 211 & 13 & 155 & 82 & 58 & 137 & 1 & 0 \\
\hline
\end{tabular}

TABLE 3: $16 \times 16$ matrix obtained after $2^{\text {nd }}$ step.

\begin{tabular}{|c|c|c|c|c|c|c|c|c|c|c|c|c|c|c|c|}
\hline 4 & 8 & 16 & 241 & 121 & 148 & 21 & 87 & 53 & 165 & 2 & 116 & 174 & 39 & 91 & 106 \\
\hline 104 & 77 & 43 & 222 & 145 & 243 & 181 & 65 & 54 & 5 & 80 & 99 & 191 & 237 & 168 & 31 \\
\hline 112 & 110 & 200 & 72 & 153 & 226 & 66 & 20 & 158 & 177 & 252 & 203 & 76 & 192 & 67 & 14 \\
\hline 251 & 70 & 103 & 49 & 115 & 119 & 246 & 3 & 159 & 63 & 12 & 117 & 233 & 48 & 19 & 196 \\
\hline 146 & 107 & 193 & 90 & 132 & 97 & 149 & 127 & 134 & 28 & 69 & 143 & 71 & 81 & 156 & 227 \\
\hline 68 & 128 & 202 & 60 & 175 & 102 & 244 & 46 & 85 & 44 & 118 & 133 & 183 & 47 & 157 & 221 \\
\hline 236 & 136 & 120 & 199 & 249 & 166 & 253 & 151 & 218 & 83 & 141 & 255 & 92 & 204 & 109 & 170 \\
\hline 207 & 64 & 150 & 101 & 40 & 59 & 247 & 152 & 220 & 224 & 239 & 144 & 34 & 52 & 167 & 84 \\
\hline 22 & 171 & 178 & 195 & 95 & 228 & 201 & 216 & 51 & 250 & 122 & 219 & 23 & 161 & 27 & 131 \\
\hline 125 & 154 & 187 & 238 & 111 & 30 & 186 & 176 & 114 & 188 & 229 & 123 & 108 & 130 & 208 & 160 \\
\hline 142 & 214 & 180 & 89 & 6 & 61 & 24 & 209 & 140 & 245 & 194 & 98 & 11 & 254 & 35 & 138 \\
\hline 212 & 32 & 225 & 75 & 163 & 248 & 169 & 26 & 17 & 164 & 42 & 242 & 232 & 73 & 182 & 78 \\
\hline 94 & 57 & 147 & 190 & 45 & 179 & 25 & 184 & 15 & 215 & 93 & 240 & 88 & 231 & 185 & 9 \\
\hline 217 & 79 & 86 & 230 & 50 & 173 & 223 & 205 & 113 & 18 & 33 & 37 & 10 & 105 & 62 & 198 \\
\hline 162 & 55 & 129 & 100 & 235 & 126 & 234 & 96 & 38 & 135 & 7 & 206 & 56 & 41 & 197 & 29 \\
\hline 82 & 137 & 1 & 0 & 189 & 36 & 74 & 210 & 124 & 139 & 213 & 172 & 13 & 211 & 58 & 155 \\
\hline
\end{tabular}

event. Let us denote the input and output masks by $T_{x}$ and $T_{y}$, respectively. Then mathematically, linear approximation probability is defined as follows.

$$
L P=\max _{T_{x}, T_{y} \neq 0}\left|\frac{\#\left\{x \in I / x \cdot T_{x}=S(x) \cdot T_{y}\right\}}{2^{n}}-\frac{1}{2}\right|
$$

In above expression $I$ denotes the set of all possible values in domain and $2^{n}$ is the number of elements of the S-box.

The maximum LP value is 0.0625 , which is matching with the best known S-boxes such as Gray, APA, and AES. In Table 9, a comparison of the results of this analysis, between our S-box and some famous S-boxes, is given.
3.5. Differential Uniformity. Differential uniformity is another important method of block cipher cryptanalysis. It was introduced by Biham and Shamir to break block ciphers [3]. It exploits certain events of $\mathrm{I} / \mathrm{O}$ differences and represents the maximum likelihood of generating an output differential $\Delta k=K_{i} \oplus K_{j}$ when the input differential is $\Delta h=H_{i} \oplus H_{j}$. In this analysis, the XOR distribution between the inputs and outputs of substitution box is computed. Mathematically, it is defined as

$$
D U=\left[\#\left\{\frac{h \varepsilon H}{S(h)} \bigoplus S(h \bigoplus \Delta h)=\Delta k\right\}\right]
$$

where \# denotes cardinality and $H$ is set of all inputs $h[3,24$, 25]. By using the approach introduced in [3], an input/output 
TABle 4: Proposed S-box evolved after $3^{\text {rd }}$ step.

\begin{tabular}{|c|c|c|c|c|c|c|c|c|c|c|c|c|c|c|c|}
\hline 142 & 125 & 220 & 89 & 219 & 63 & 251 & 158 & 149 & 46 & 126 & 146 & 28 & 208 & 144 & 218 \\
\hline 245 & 9 & 189 & 17 & 120 & 240 & 159 & 166 & 79 & 165 & 128 & 73 & 241 & 26 & 137 & 7 \\
\hline 118 & 83 & 78 & 99 & 228 & 21 & 138 & 183 & 1 & 246 & 117 & 170 & 217 & 207 & 60 & 75 \\
\hline 145 & 231 & 171 & 22 & 55 & 39 & 242 & 154 & 134 & 199 & 56 & 213 & 214 & 11 & 147 & 53 \\
\hline 255 & 148 & 41 & 62 & 71 & 244 & 197 & 203 & 133 & 100 & 30 & 188 & 185 & 140 & 93 & 253 \\
\hline 172 & 69 & 119 & 151 & 12 & 180 & 139 & 57 & 233 & 65 & 35 & 111 & 43 & 238 & 132 & 66 \\
\hline 20 & 77 & 201 & 173 & 84 & 155 & 91 & 179 & 74 & 32 & 193 & 176 & 29 & 164 & 80 & 113 \\
\hline 59 & 235 & 136 & 52 & 64 & 175 & 3 & 192 & 19 & 186 & 156 & 88 & 6 & 169 & 61 & 110 \\
\hline 51 & 243 & 14 & 18 & 227 & 101 & 121 & 58 & 191 & 143 & 45 & 114 & 225 & 152 & 254 & 153 \\
\hline 24 & 48 & 222 & 70 & 105 & 50 & 206 & 25 & 72 & 127 & 67 & 5 & 112 & 215 & 90 & 96 \\
\hline 135 & 181 & 195 & 16 & 194 & 174 & 92 & 36 & 10 & 210 & 236 & 130 & 216 & 40 & 86 & 248 \\
\hline 239 & 229 & 54 & 102 & 33 & 212 & 44 & 129 & 161 & 184 & 205 & 226 & 34 & 187 & 202 & 0 \\
\hline 182 & 178 & 232 & 42 & 106 & 190 & 204 & 87 & 122 & 103 & 49 & 107 & 15 & 249 & 124 & 234 \\
\hline 163 & 141 & 37 & 237 & 211 & 209 & 221 & 38 & 250 & 198 & 115 & 85 & 162 & 68 & 108 & 224 \\
\hline 4 & 167 & 2 & 95 & 247 & 109 & 196 & 252 & 13 & 98 & 104 & 8 & 116 & 223 & 160 & 177 \\
\hline 230 & 23 & 168 & 131 & 47 & 123 & 27 & 82 & 31 & 97 & 76 & 157 & 200 & 150 & 81 & 94 \\
\hline
\end{tabular}

TABLE 5: The nonlinearity test outcomes of different substitution boxes.

\begin{tabular}{|c|c|c|c|c|c|c|c|c|c|}
\hline S boxes & 0 & 1 & 2 & 3 & 4 & 5 & 6 & 7 & Ave \\
\hline Suggested S-box & 112 & 112 & 112 & 112 & 112 & 112 & 112 & 112 & 112 \\
\hline Coset Diagram S-box [7] & 108 & 106 & 108 & 108 & 108 & 104 & 106 & 106 & 106.75 \\
\hline Gray [8] & 112 & 112 & 112 & 112 & 112 & 112 & 112 & 112 & 112 \\
\hline Arun [9] & 108 & 106 & 104 & 98 & 102 & 102 & 98 & 74 & 99 \\
\hline Prime $[10]$ & 94 & 100 & 104 & 104 & 102 & 100 & 98 & 94 & 99.5 \\
\hline $\mathrm{S}_{8}$ AES [11] & 112 & 112 & 112 & 112 & 112 & 112 & 112 & 112 & 112 \\
\hline Xyi [12] & 106 & 104 & 106 & 106 & 104 & 106 & 104 & 106 & 105 \\
\hline AES [6] & 112 & 112 & 112 & 112 & 112 & 112 & 112 & 112 & 112 \\
\hline Skipjack [13] & 104 & 108 & 108 & 108 & 108 & 104 & 104 & 106 & 106.75 \\
\hline Alkhaldi [14] & 108 & 104 & 106 & 106 & 102 & 98 & 104 & 108 & 104 \\
\hline Chen [15] & 100 & 102 & 103 & 104 & 106 & 106 & 106 & 108 & 104.3 \\
\hline Tang [16] & 100 & 103 & 104 & 104 & 105 & 105 & 106 & 109 & 104.5 \\
\hline Khan [17] & 102 & 108 & 106 & 102 & 106 & 106 & 106 & 98 & 104.25 \\
\hline Belazi [18] & 106 & 106 & 106 & 104 & 108 & 102 & 106 & 104 & 105.25 \\
\hline
\end{tabular}

XOR distribution matrix of size $16 \times 16$ is calculated for suggested S-box and is provided in Table 10. As a general Sbox design guideline, the maximum differential uniformity has to be kept as low as possible to withstand differential attacks. The highest value of differential uniformity for suggested S-box is 4, which is compared with some wellknown S-boxes in Table 11 to show the strength of suggested S-box.

\section{Majority Logic Criterion}

In majority logic criterion, statistical analyses are performed to examine the statistical strength of the S-box in image encryption application [26]. The encryption process creates a distortion in the image, these kinds of distortions determine the strength of the algorithm. Therefore, it is necessary to investigate the statistical properties through various analyses.
These analyses are correlation, entropy, contrast, homogeneity, and energy. The suggested S-boxes can further be used for encryption and multimedia security. We have used two JPEG images, Pepper and Baboon, for MLC analysis. The results of these analyses in comparison with the other well-known Sboxes are depicted in Table 12. Figure 2 shows the result of image encryption with proposed S-box. The histograms of the original image and the encrypted images of Baboon and Pepper are shown in Figure 3. These results indicate that the proposed S-box is suitable for encryption applications and is adequate enough to become part of the algorithms designed for the secure transmission of information/data.

\section{Conclusion}

In this study, we introduce a group theoretic technique to form strong S-boxes. The cyclic group $C_{255}$ instead of a Galois 
TABLE 6: BIC nonlinearity for the suggested S-box.

\begin{tabular}{lcccccccc}
\hline Rows/Columns & 0 & 1 & 2 & 3 & 4 & 5 & 6 & 712 \\
\hline 0 & - & 112 & 112 & 112 & 112 & 112 & 112 \\
1 & 112 & - & 112 & 112 & 112 & 112 & 112 \\
2 & 112 & 112 & - & 112 & 112 & 112 & 112 \\
3 & 112 & 112 & 112 & - & 112 & 112 & 112 \\
4 & 112 & 112 & 112 & 112 & - & 112 & 112 \\
5 & 112 & 112 & 112 & 112 & 112 & - & 112 \\
6 & 112 & 112 & 112 & 112 & 112 & 112 & - \\
7 & 112 & 112 & 112 & 112 & 112 & 112 & 112 \\
\hline
\end{tabular}

TABLE 7: BIC results of different S-boxes.

\begin{tabular}{lccc}
\hline S-boxes & Minimum value & Average & Square deviation \\
\hline Suggested S-box & 112 & 112 & 0 \\
Gray & 112 & 112 & 0 \\
Arun & 92 & 103 & 3.5225 \\
Prime & 94 & 101.71 & 3.53 \\
S $_{8}$ AES & 112 & 112 & 0 \\
Xyi & 98 & 103.78 & 2.743 \\
AES & 112 & 112 & 0 \\
Skipjack & 102 & 104.14 & 1.767 \\
\hline
\end{tabular}

TABLE 8: Values of SAC for the suggested S-box.

\begin{tabular}{lcccccccc}
\hline Rows/Columns & 0 & 1 & 2 & 3 & 4 & 5 & 6 \\
\hline 0 & 0.4844 & 0.4688 & 0.4844 & 0.5469 & 0.4688 & 0.5625 & 0.5469 & 0.5313 \\
1 & 0.4531 & 0.5313 & 0.5156 & 0.5469 & 0.4688 & 0.4688 & 0.4844 & 0.5469 \\
2 & 0.5000 & 0.4688 & 0.4609 & 0.4688 & 0.5156 & 0.4531 & 0.5469 & 0.5625 \\
3 & 0.5313 & 0.5234 & 0.5313 & 0.5156 & 0.4531 & 0.4688 & 0.5234 & 0.4375 \\
4 & 0.5625 & 0.4844 & 0.4688 & 0.5156 & 0.5469 & 0.5469 & 0.5625 & 0.4844 \\
5 & 0.5000 & 0.4844 & 0.5156 & 0.5625 & 0.4844 & 0.5469 & 0.4844 & 0.5156 \\
6 & 0.4844 & 0.5156 & 0.5000 & 0.4844 & 0.4844 & 0.4844 & 0.4688 & 0.5625 \\
7 & 0.5469 & 0.5625 & 0.4531 & 0.4688 & 0.5156 & 0.4844 & 0.5313 & 0.4844 \\
\hline
\end{tabular}

TABLE 9: Linear approximation probability analyses of different S-boxes.

\begin{tabular}{lcccccccc}
\hline S-boxes & Suggested S-box & AES & Skipjack & Prime & Gray & Arun & S $_{8}$ AES & Xyi \\
\hline Max value & 144 & 144 & 156 & 162 & 144 & 164 & 144 & 168 \\
Max LP & 0.062 & 0.062 & 0.109 & 0.132 & 0.062 & 0.2109 & 0.062 & 0.156 \\
\hline
\end{tabular}

field is used to destroy the initial sequence $0,1,2, \ldots, 255$. The construction of S-box involves three simple steps:

(i) First present the elements of $C_{255} \cup\{0\}=$ $\left\{T(1), T^{2}(1), T^{3}(1), \ldots, T^{254}(1), 1,0\right\}$ in $16 \times 16$ matrix.

(ii) Next, apply two permutations of $S_{16}$ on rows and column of the matrix. It will significantly improve the performance of the S-box.

(iii) In the last step, a permutation of $S_{256}$ is applied on the matrix (obtained in step (ii)) to form proposed S-box.
The results acquired from different analyses show that the performance of our S-box against various algebraic attacks is much better than most of well-known S-boxes and similar to AES, $S_{8}$ AES, and Gray S-boxes. Therefore, our S-box meets all the requirements and is considered as a strong S-box for the secure communication.

\section{Data Availability}

The data used to support the findings of this study are available from the corresponding author upon request. 
TABLE 10: Differential uniformity of proposed S-box.

\begin{tabular}{|c|c|c|c|c|c|c|c|c|c|c|c|c|c|c|c|}
\hline 4 & 4 & 4 & 4 & 4 & 4 & 4 & 4 & 4 & 4 & 4 & 4 & 4 & 4 & 4 & 4 \\
\hline 4 & 4 & 4 & 4 & 4 & 4 & 4 & 4 & 4 & 4 & 4 & 4 & 4 & 4 & 4 & 4 \\
\hline 4 & 4 & 4 & 4 & 4 & 4 & 4 & 4 & 4 & 4 & 4 & 4 & 4 & 4 & 4 & 4 \\
\hline 4 & 4 & 4 & 4 & 4 & 4 & 4 & 4 & 4 & 4 & 4 & 4 & 4 & 4 & 4 & 4 \\
\hline 4 & 4 & 4 & 4 & 4 & 4 & 4 & 4 & 4 & 4 & 4 & 4 & 4 & 4 & 4 & 4 \\
\hline 4 & 4 & 4 & 4 & 4 & 4 & 4 & 4 & 4 & 4 & 4 & 4 & 4 & 4 & 4 & 4 \\
\hline 4 & 4 & 4 & 4 & 4 & 4 & 4 & 4 & 4 & 4 & 4 & 4 & 4 & 4 & 4 & 4 \\
\hline 4 & 4 & 4 & 4 & 4 & 4 & 4 & 4 & 4 & 4 & 4 & 4 & 4 & 4 & 4 & 4 \\
\hline 4 & 4 & 4 & 4 & 4 & 4 & 4 & 4 & 4 & 4 & 4 & 4 & 4 & 4 & 4 & 4 \\
\hline 4 & 4 & 4 & 4 & 4 & 4 & 4 & 4 & 4 & 4 & 4 & 4 & 4 & 4 & 4 & 4 \\
\hline 4 & 4 & 4 & 4 & 4 & 4 & 4 & 4 & 4 & 4 & 4 & 4 & 4 & 4 & 4 & 4 \\
\hline 4 & 4 & 4 & 4 & 4 & 4 & 4 & 4 & 4 & 4 & 4 & 4 & 4 & 4 & 4 & 4 \\
\hline 4 & 4 & 4 & 4 & 4 & 4 & 4 & 4 & 4 & 4 & 4 & 4 & 4 & 4 & 4 & 4 \\
\hline 4 & 4 & 4 & 4 & 4 & 4 & 4 & 4 & 4 & 4 & 4 & 4 & 4 & 4 & 4 & 4 \\
\hline 4 & 4 & 4 & 4 & 4 & 4 & 4 & 4 & 4 & 4 & 4 & 4 & 4 & 4 & 4 & 4 \\
\hline 4 & 4 & 4 & 4 & 4 & 4 & 4 & 4 & 4 & 4 & 4 & 4 & 4 & 4 & 4 & - - \\
\hline
\end{tabular}

TABLE 11: Maximum differential uniformity of various S-boxes.

\begin{tabular}{lcccccccccc}
\hline S-boxes & Suggested S-box & AES & Gray & Skipjack & Chen & Khan & S $_{8}$ AES & Tang & Xyi \\
\hline Max DU & 4 & 4 & 4 & 12 & 12 & 16 & 4 & 10 & 12 \\
\hline
\end{tabular}
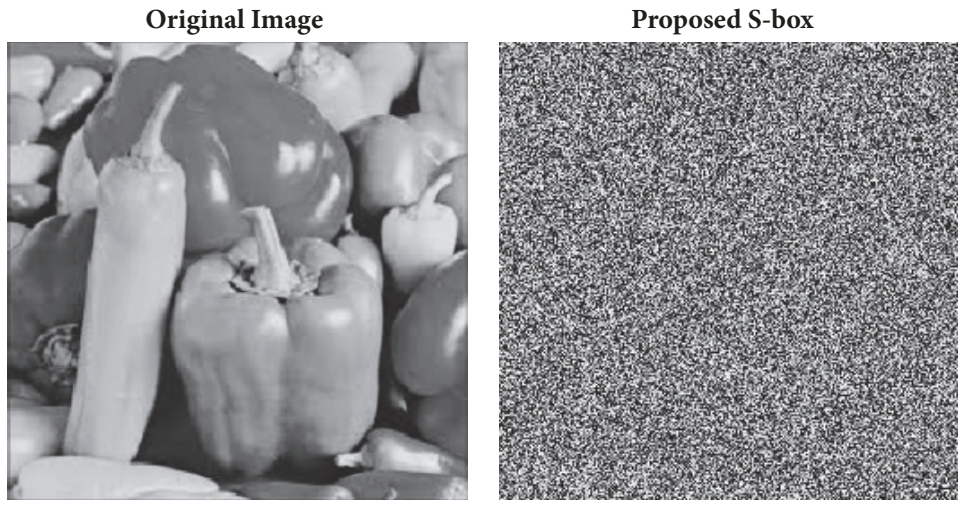

(a)
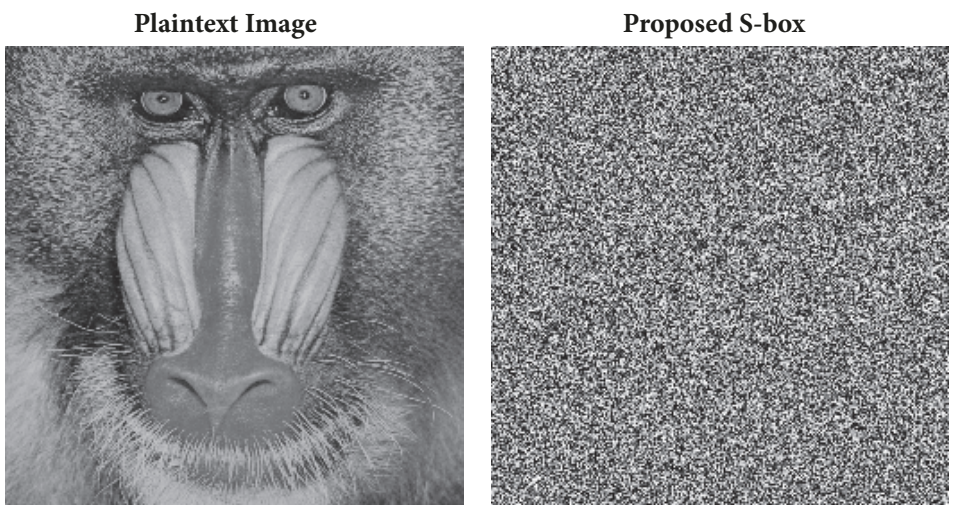

(b)

FIgURE 2: Original image and the encrypted images using two rounds of encryption: (a) Pepper and (b) Baboon. 

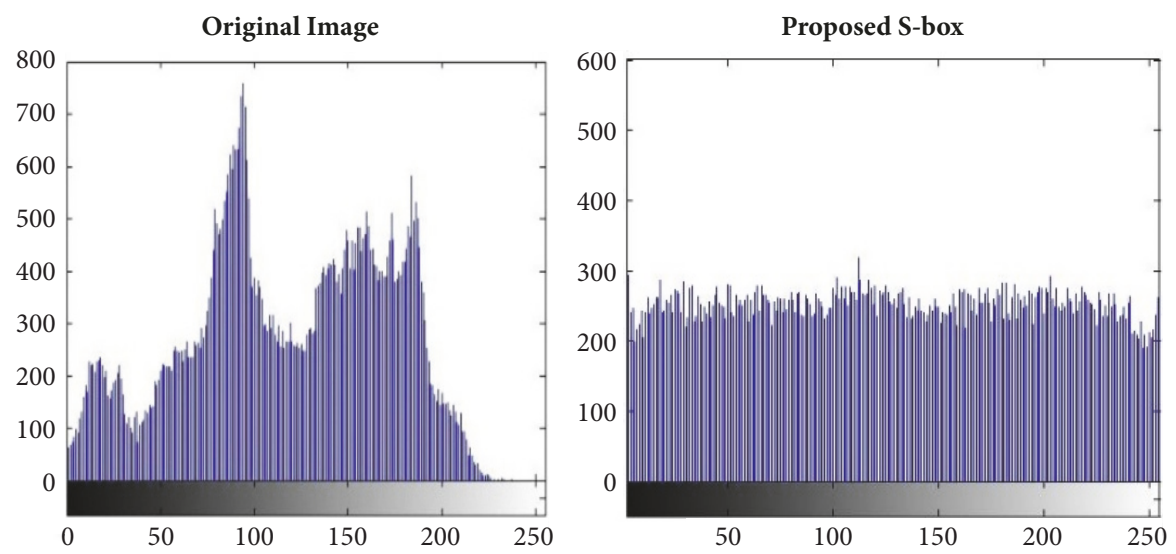

(a)
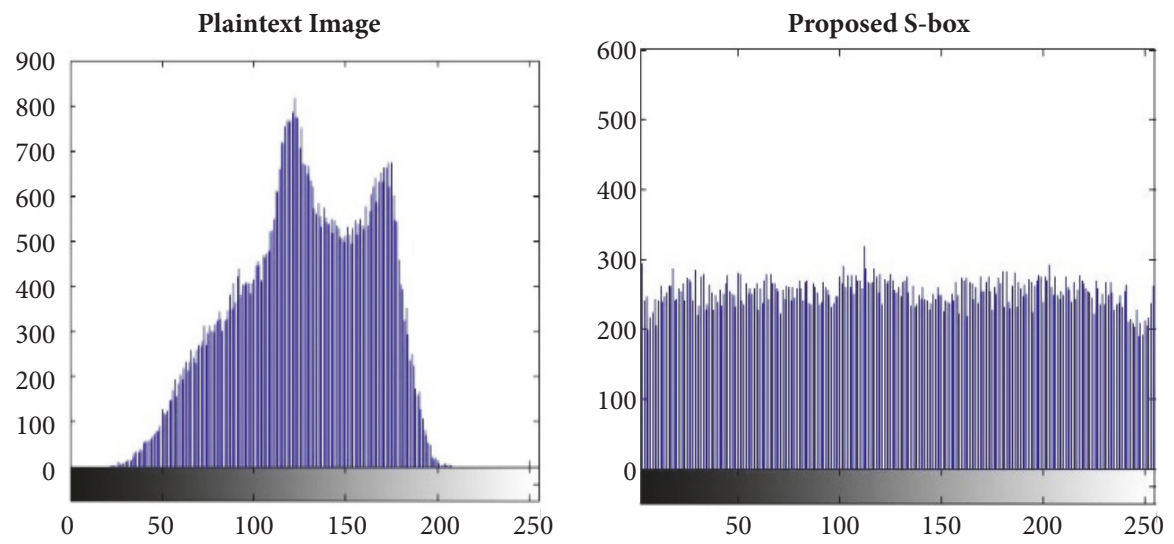

(b)

Figure 3: Histogram of the original image and the encrypted images: (a) Pepper and (b) Baboon.

TABLE 12: Comparison of Majority logic criterion for S-box over various S-boxes.

\begin{tabular}{|c|c|c|c|c|c|}
\hline S-boxes & Correlation & Entropy & Contrast & Homogeneity & Energy \\
\hline \multicolumn{6}{|l|}{ Pepper Image } \\
\hline Plain Text & 0.9383 & 7.5909 & 0.2760 & 0.9024 & 0.1288 \\
\hline Suggested S-box & -0.0134 & 7.9842 & 8.6969 & 0.4045 & 0.0174 \\
\hline Atta [19] & -0.0043 & 7.9823 & 8.6727 & 0.4076 & 0.0173 \\
\hline Skipjack & 0.1205 & 7.7561 & 7.7058 & 0.4708 & 0.0239 \\
\hline Khan [20] & 0.0103 & 7.9562 & 8.3129 & 0.4219 & 0.0180 \\
\hline Belazi & -0.0112 & 7.9233 & 8.1423 & 0.4648 & 0.0286 \\
\hline \multicolumn{6}{|l|}{ Baboon Image } \\
\hline Plain Text & 0.6782 & 7.1273 & 0.7179 & 0.7669 & 0.1025 \\
\hline Suggested S-box & -0.0060 & 7.9820 & 8.6488 & 0.4062 & 0.0174 \\
\hline AES & 0.0554 & 7.2531 & 7.5509 & 0.4662 & 0.0202 \\
\hline Prime & 0.0855 & 6.9311 & 7.6236 & 0.4640 & 0.0202 \\
\hline Xyi & 0.0417 & 7.2531 & 8.3108 & 0.4533 & 0.0196 \\
\hline Skipjack & 0.1025 & 7.2531 & 7.7058 & 0.4689 & 0.0193 \\
\hline Khan [14] & -0.0512 & 7.9612 & 8.1213 & 0.4011 & 0.0210 \\
\hline Belazi & 0.01190 & 7.9252 & 8.0391 & 0.4428 & .02219 \\
\hline
\end{tabular}




\section{Conflicts of Interest}

The authors declare that there are no conflicts of interest regarding the publication of this paper.

\section{Acknowledgments}

This research project was supported by a grant from the Research Center of the Center for Female Scientific and Medical Colleges, Deanship of Scientific Research, King Saud University.

\section{References}

[1] C. E. Shannon, "Communication theory of secrecy systems," Bell Labs Technical Journal, vol. 28, no. 4, pp. 656-715, 1949.

[2] L. R. Knudsen and M. J. B. Robshaw, The Block Cipher Companion, Springer, Berlin, 2011.

[3] E. Biham and A. Shamir, "Differential cryptanalysis of DES-like cryptosystems," Journal of Cryptology, vol. 4, no. 1, pp. 3-72, 1991.

[4] T. W. Cusick and P. Stanica, Cryptographic Boolean functions and applications, Academic Press, San Diego, CA, USA, 2009.

[5] T. Helleseth, "Linear cryptanalysis method for des cipher," in Advances in Cryptology-EUROCRYPT, vol. 765 of Lecture Notes in Computer Science, pp. 386-397, Springer, Berlin, Germany, 1993.

[6] J. Daemen and V. Rijmen, The design of Rijndael-AES: the advanced encryption standard, Springer, Berlin, 2002.

[7] A. Razaq, A. Yousaf, U. Shuaib, N. Siddiqui, A. Ullah, and A. Waheed, "A Novel Construction of Substitution Box Involving Coset Diagram and a Bijective Map," Security and Communication Networks, vol. 2017, 2017.

[8] M. T. Tran, D. K. Bui, and A. D. Doung, "Gray S-box for advanced encryption standard," in Proceedings of the International Conference on Computer Intel Security, vol. 1, pp. 253-258, 2008.

[9] A. Gautam, G. S. Gaba, R. Miglani, and R. Pasricha, "Application of Chaotic Functions for Construction of Strong Substitution Boxes," Indian Journal of Science and Technology, vol. 8, no. 28, pp. 1-5, 2015.

[10] I. Hussain, T. Shah, H. Mahmood, M. A. Gondal, and U. Y. Bhatti, "Some analysis of S-box based on residue of prime number," Proceedings of the Pakistan Academy of Sciences, vol. 48, no. 2, pp. 111-115, 2011.

[11] I. Hussain, T. Shah, and H. Mahmood, "A new algorithm to construct secure keys for AES," International Journal of Contemporary Mathematical Sciences, vol. 5, no. 25-28, pp. 1263-1270, 2010.

[12] X. Y. Shi, Hu. Xiao, X. C. You, and K. Y. Lam, "A method for obtaining cryptographically strong $8^{\star} 8$ S-boxes," in Proceedings of the International Conference on Advanced Information Networking and Applications, vol. 2, pp. 14-20, 2002.

[13] Skipjack and Kea: Algorithm Specifications Version, 1998, http:// csrc.nist.gov/CryptoToolkit/.

[14] A. H. Alkhaldi, I. Hussain, and M. A. Gondal, "A novel design for the construction of safe S-boxes based on TDERC sequence," Alexandria Engineering Journal, vol. 54, pp. 65-69, 2015.

[15] G. Chen, Y. Chen, and X. Liao, "An extended method for obtaining S-boxes based on three-dimensional chaotic baker maps," Chaos, Solitons \& Fractals, vol. 31, no. 3, pp. 571-579, 2007.

[16] G. Tang, X. Liao, and Y. Chen, "A novel method for designing S-boxes based on chaotic maps," Chaos, Solitons \& Fractals, vol. 23, no. 2, pp. 413-419, 2005.

[17] M. Khan, T. Shah, and M. A. Gondal, "An efficient technique for the construction of substitution box with chaotic partial differential equation," Nonlinear Dynamics, vol. 73, no. 3, pp. 1795-1801, 2013.

[18] A. Belazi, M. Khan, A. A. A. El-Latif, and S. Belghith, "Efficient cryptosystem approaches: S-boxes and permutationsubstitution-based encryption," Nonlinear Dynamics, vol. 87, no. 1, pp. 337-361, 2017.

[19] A. Ullah, S. S. Jamal, and T. Shah, "A novel construction of substitution box using a combination of chaotic maps with improved chaotic range," Nonlinear Dynamics, vol. 88, no. 4, pp. 2757-2769, 2017

[20] M. Khan, T. Shah, and S. I. Batool, "Construction of S-box based on chaotic Boolean functions and its application in image encryption," Neural Computing and Applications, vol. 27, no. 3, pp. 677-685, 2016.

[21] G. V. Bard, Algebraic Cryptanalysis, Springer, Berlin, 2009.

[22] A. Webster and S. Tavares, "On the design of S-boxes," in Advancesin Cryptology: Proc. of Crypto'85 Lecture Notes in Computer Science, pp. 523-534, 1986.

[23] J. Pieprzyk and G. Finkelstein, "Towards effective nonlinear cryptosystem design," IEE Proceedings Part E Computers and Digital Techniques, vol. 135, no. 6, pp. 325-335, 1988.

[24] H. A. Ahmed, M. F. Zolkipli, and M. Ahmad, "A novel efficient substitution-box design based on firefly algorithm and discrete chaotic map," Neural Computing and Applications, pp. 1-10.

[25] E. Al Solami, M. Ahmad, C. Volos, M. Doja, and M. Beg, "A New Hyperchaotic System-Based Design for Efficient Bijective Substitution-Boxes," Entropy, vol. 20, no. 7, p. 525, 2018.

[26] I. Hussain, T. Shah, M. A. Gondal, and H. Mahmood, "Generalized Majority Logic Criterion to Analyze the Statistical Strength of S-Boxes," Zeitschrift für Naturforschung A, vol. 67, no. 5, pp. 282-288, 2012. 


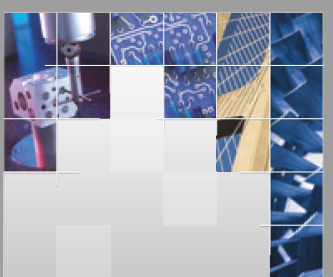

\section{Enfincering}
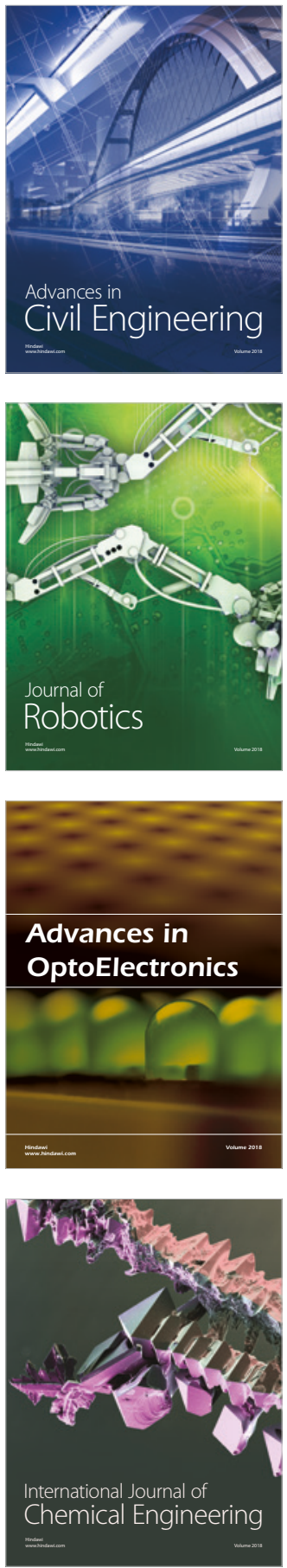

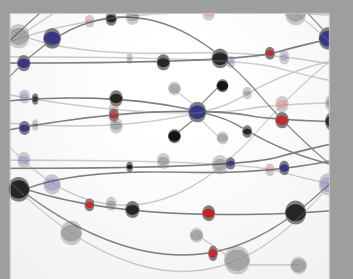

\section{Rotating \\ Machinery}

The Scientific World Journal

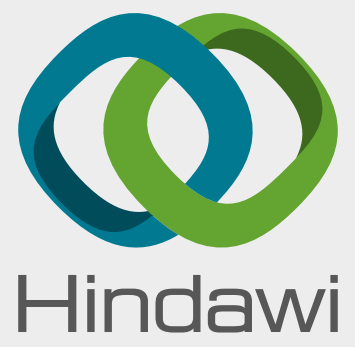

Submit your manuscripts at

www.hindawi.com
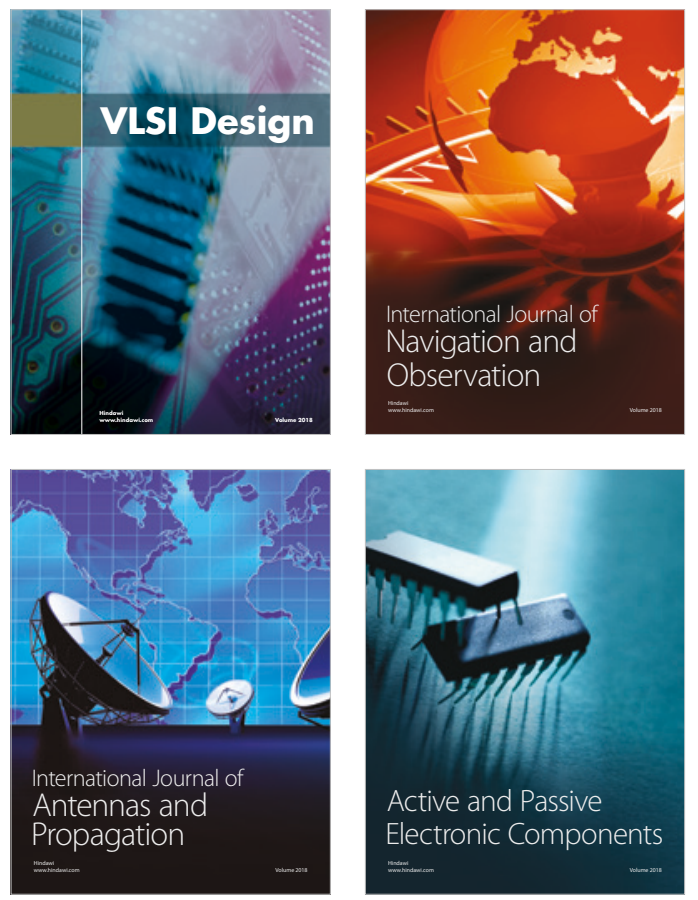
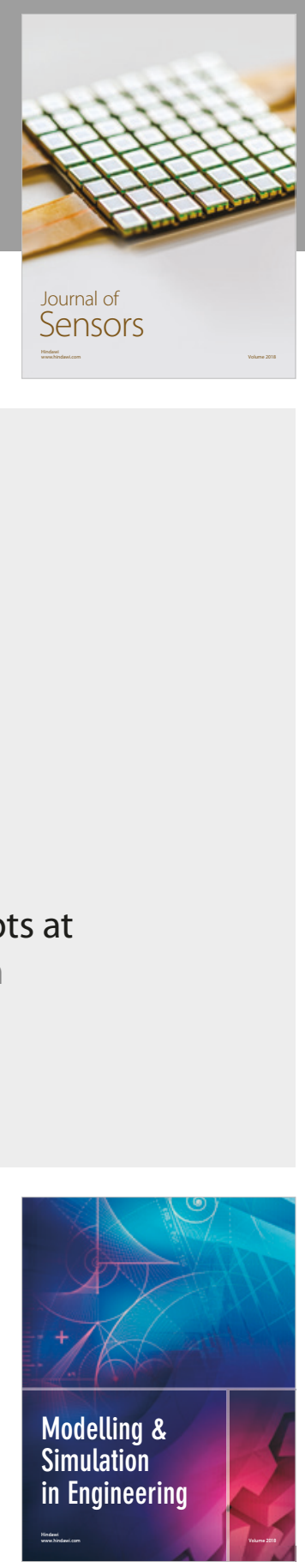

\section{Advances \\ Multimedia}
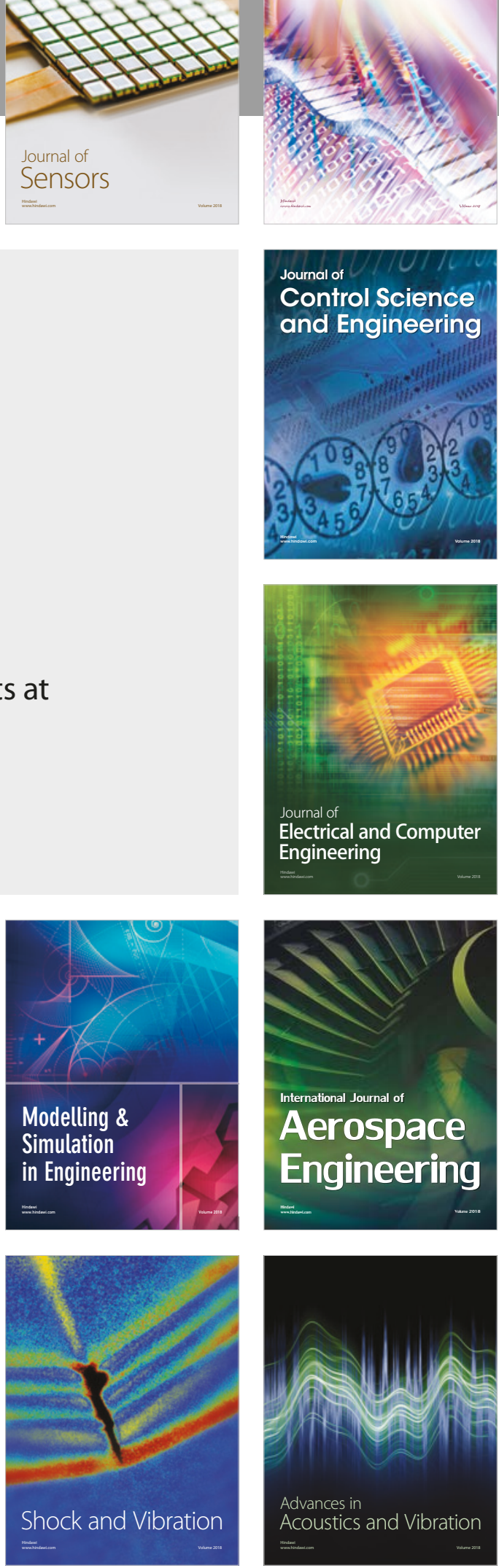